\title{
Comportamento da criança perante a presença das mães durante a assistência odontológica
}

\author{
The child's behavior before the presence of mothers during dental care \\ El comportamiento del niño en presencia de las madres durante el cuidado dental
}

\author{
Giovanna Rodrigues MENESES ${ }^{1}$ \\ Martha Suemi SAKASHITA \\ Raquel Carros ANTONIO ${ }^{2}$ \\ Valéria Cristina Lopes de Barros ROLIM ${ }^{2}$ \\ Adriana Sales CUNHA-CORREIA ${ }^{2}$ \\ ${ }^{1}$ Graduanda de Odontologia, Faculdade de Odontologia da Universidade Brasil - 15600-000 Fernandópolis-SP, Brasil \\ ${ }^{2}$ Disciplina de Clínica Odontológica Integrada Infantil, \\ Faculdade de Odontologia da Universidade Brasil - 15600-000 Fernandópolis-SP, Brasil
}

\begin{abstract}
Resumo
Introdução: em Odontopediatria, o medo e a ansiedade são fatores que podem prejudicar a assistência, uma vez que o manejo do comportamento infantil, bem como o controle da ansiedade das mães pode ser uma tarefa difícil para o profissional. Objetivo: o objetivo deste estudo foi realizar uma revisão da literatura sobre como a presença e comportamento das mães na sala de atendimento pode influenciar o comportamento de seus filhos durante a assistência odontológica. Material e método: esta revisão foi elaborada a partir da busca bibliográfica de artigos científicos publicados em periódicos nacionais e internacionais indexados nas bases de dados Lilacs, Medline e PubMed. Resultados: foram encontrados 26 artigos científicos publicados nas línguas portuguesa e inglesa. A maioria dos autores acredita que não há relevante alteração de comportamento da criança perante a presença das mães, e que grande parte dos responsáveis prefere estar presente na sala de atendimento. Conclusão: Concluiu-se que seria favorável que o paciente fizesse a primeira visita ao consultório odontológico o mais cedo possível, para que ele se acostume com o ambiente e os procedimentos, o medo e a ansiedade sejam controlados por métodos e técnicas, havendo ainda o diálogo e participação dos pais, proporcionando uma adequada relação do profissional com o paciente e sua família. As mães não são a maior influência no comportamento inadequado da criança durante a assistência odontológica, podendo inclusive auxiliar o profissional no manejo do comportamento infantil dentro ou fora da sala de atendimento.

Descritores: Comportamento Materno; Comportamento Infantil; Relações Dentista-Paciente.
\end{abstract}

\begin{abstract}
Introduction: in Pediatric Dentistry, fear and anxiety are factors that can harm the assistance, since the management of children's behavior, as well as control of the anxiety of mothers can be a difficult task for the professional. Objective: the aim of this study was to review the literature on the presence and behavior of mothers in the waiting room can influence the behavior of their children during dental care. Method: this review was drawn from the literature search of scientific articles published in national and international journals indexed in databases Lilacs, Medline and PubMed. Results: 26 scientific articles published in Portuguese and English were found. Most authors believe that there is no relevant child behavior change before the presence of mothers, and that most of those responsible prefer to be present in the consulting room. Conclusion: it was concluded that it would be favorable that the patient was the first visit to the dental office as soon as possible, so that they get used to the environment and procedures, fear and anxiety are controlled by methods and techniques, yet there is dialogue and parental involvement, providing appropriate professional relationship with the patient and his family. Mothers are not the major influence on the child's inappropriate behavior during dental care, and may even assist the professional in the management of child behavior inside or outside the care room.

Descriptors: Maternal Behavior; Child Behavior; Dentist-Patient Relations.
\end{abstract}

\section{Resumen}

Introducción: en odontología pediátrica, el miedo y la ansiedad son factores que pueden dañar la ayuda, ya que el manejo de la conducta de los niños, así como el control de la ansiedad de las madres puede ser una tarea difícil para el profesional. Objetivo: el objetivo de este estudio fue revisar la literatura sobre la presencia y el comportamiento de las madres en la sala de espera puede influir en el comportamiento de sus hijos durante la atención dental. Método: este informe ha sido realizado a partir de la búsqueda bibliográfica de artículos científicos publicados en revistas nacionales e internacionales indexadas en bases de datos Lilacs, Medline y PubMed. Resultados: se encontraron 26 artículos científicos publicados en portugués y en Inglés. La mayoría de los autores creen que no hay cambio en el comportamiento del niño pertinente ante la presencia de las madres, y que la mayoría de los responsables prefieren estar presente en la sala de consulta. Conclusión: se concluyó que sería favorable que el paciente fue la primera visita al consultorio dental tan pronto como sea posible, para que se acostumbren al medio ambiente y los procedimientos, el miedo y la ansiedad son controlados por los métodos y técnicas, sin embargo, no es el diálogo y la participación de los padres, proporcionando relación profesional adecuada con el paciente y su familia. Las madres no son la mayor influencia en el comportamiento inadecuado del niño durante el cuidado dental e incluso pueden ayudar al profesional en la gestión de la conducta de los niños dentro o fuera de la sala de consulta.

Descriptores: Comportamiento Maternal; Comportamiento Del Niño; Relación Dentista-Paciente.

\section{INTRODUÇÃO}

A Odontopediatria, juntamente com o desenvolvimento adequado da saúde bucal das crianças, ajuda a fornecer uma perspectiva positiva a elas quanto a visitar o dentista. Assim sendo, deve-se tentar controlar a ansiedade e o medo das crianças, utilizando diferentes técnicas de manejo ${ }^{1}$. De acordo com Beaton et al. ${ }^{2}$, metade 
das pessoas que foram ao dentista informou que seu medo começou na infância. Esta relação entre a ansiedade de ir ao dentista e início da infância reforça a necessidade de compreender a etiologia de medo de dentista, a fim de impedir esse potencial para a doença na idade adulta.

As situações mais difíceis enfrentadas pelos odontopediatras são aquelas em que as crianças, principalmente as mais novas, não colaboram com a realização do tratamento, chorando, gritando, movimentando a cabeça e o corpo, e fazendo tentativas de sair da cadeira. Esses comportamentos de não colaboração são, geralmente, atribuídos ao medo, a traumas, a condições fisiológicas ou a outros fatores inerentes ao indivíduo, sendo dado pouco valor para os eventos presentes no ambiente odontológico ${ }^{3}$. (Brandenburg et al. ${ }^{3}$, 2009).

Para Cademartori ${ }^{4}$ a criança deve ser tratada como ser único, individual, e respeitada em suas necessidades, sendo que o Odontopediatra deve ser capaz de perceber quando determinada situação ou fator possa estar ocasionando modificações no seu comportamento. A autora relata a importância de reconhecer este estado de emoção da criança e, os preditores do comportamento infantil, pois além de facilitar a rotina de atendimento, isso permitirá que o profissional lide mais facilmente com as situações adversas sem torná-las traumáticas ou provocadoras de sofrimento. Já Losso et al. ${ }^{5}$ acreditam que os dentistas devem considerar a etiologia multifatorial da ansiedade para obter uma melhor compreensão dos pacientes pediátricos e suas famílias, e que para oferecer atendimento odontológico mais confortável, a prevalência de ansiedade de ir ao dentista não deve ser negligenciada na prática clínica, especialmente em odontopediatria.

De acordo com Goettems et al. ${ }^{6}$, os pais têm responsabilidade pela saúde bucal de seus filhos, e a ansiedade dos pais pode influenciar atitudes e hábitos de seus filhos na hora de cuidar de sua saúde bucal. Em 2010 Araújo et al. ${ }^{7}$ realizaram estudo a fim de conhecer a opinião dos pais a respeito da sua permanência junto à criança durante o atendimento odontológico, e observaram que $78,8 \%$ dos pais gostariam de estar presente durante o atendimento odontológico de seus filhos.

Com base nos aspectos apresentados, o objetivo deste estudo foi realizar uma revisão da literatura sobre como a presença e comportamento das mães na sala de atendimento pode influenciar o comportamento de seus filhos durante a assistência odontológica.

\section{MATERIAL E MÉTODO}

Esta revisão foi elaborada a partir da busca bibliográfica de artigos científicos publicados em periódicos nacionais e internacionais indexados nas bases de dados Lilacs, Medline e PubMed. Foram utilizadas neste levantamento as palavras-chave: Comportamento materno; comportamento infantil; relações dentista-paciente. Após a busca, foram encontrados 26 artigos científicos, no período de 2007 a 2016, em língua portuguesa e inglesa.

\section{REVISÃO DA LITERATURA}

Para Tomita et al. ${ }^{8}$ um aspecto crítico a ser considerado entre os trabalhos que se propõem a investigar a ansiedade materna no consultório de odontopediatria e sua relação com os comportamentos das crianças é a limitação metodológica no que se refere ao reduzido número de sessões experimentais, geralmente restritas a uma ou duas sessões, à grande quantidade de dados obtidos a partir da percepção dos pais sobre sua própria ansiedade, referida em entrevistas pré e/ou pós sessão e/ou por instrumentos de auto-avaliação, nem sempre validados e aos poucos estudos que utilizam metodologia observacional direta para identificar comportamentos indicadores de ansiedade. Os autores apontam a necessidade de se descrever e analisar funcionalmente o repertório de comportamentos das mães que acompanham seus filhos ao consultório de odontopediatria, antes de se investigar a possível relação entre esses comportamentos e as respostas apresentadas pelas crianças.

Khawja et al. ${ }^{9}$ também realizaram estudo para avaliar a ansiedade entre as mães e sua possível relação com a experiência de cárie em seus filhos na cidade de Udaipur, Índia. Quase metade $(49,7 \%)$ das mães classificou-se como sendo "bastante ansiosa" ou "muito ansiosa". Os autores concluíram que houve uma forte associação entre a ansiedade odontológica materna com a cárie infantil e que mães com baixa renda familiar apresentaram maior grau de ansiedade.

Goettems et al. ${ }^{6}$ relatam que meninos mais velhos e os filhos de família de baixa renda apresentaram maior índice de ansiedade e cárie, e sugerem que estratégias preventivas devem incidir não só sobre a criança da família, mas também sobre o comportamento materno durante o tratamento. Coric et al. ${ }^{10}$ afirmam que a evidência existente sobre o efeito dos pais sobre a DFA (uma associação entre o medo de dentista e ansiedade) de uma criança é conflitante e também que se os pais servem de modelo para o desenvolvimento da DFA, as intervenções voltadas para os pais podem ajudar a minimizar o desenvolvimento da DFA em crianças. Finalmente, os autores relatam que os cirurgiões dentistas são outra figura importante, porque eles podem controlar os acontecimentos no cenário odontológico e encorajar um comportamento mais adequado.

No estudo de Colares et al. ${ }^{11}$ foram avaliadas a ansiedade, dor e/ou desconforto durante o tratamento dentário em crianças de 5 a 12 anos de idade, sendo que a ansiedade foi medida utilizando-se o questionário de ansiedade odontológica (DAQ). Os autores concluíram que uma elevada percentagem de crianças apresentou ansiedade odontológica e história de dor de dente. Peretz et al. ${ }^{12}$ também realizaram estudo sobre a ansiedade odontológica na clínica odontopediátrica de estudantes, analisando crianças, pais e estudantes, e concluíram que a ansiedade dos alunos, ou ansiedade antes do tratamento de uma criança, pode não ser diretamente associada com a ansiedade odontológica. Os autores também relataram que uma indicação positiva entre ansiedade odontológica dos pais implica que há uma influência significativa sobre a ansiedade de seus filhos.

Em 2009, Kotsanos et al. ${ }^{13}$ realizaram uma técnica chamada PPA (pais presentes/ausentes) para gerenciar o comportamento da criança não-colaboradora. Neste estudo, 440 crianças entre 3 a 10 anos foram analisadas. Segundo a técnica, as crianças que apresentavam um comportamento "negativo" ou "definitivamente negativo", só iriam ter a presença dos pais se seu comportamento fosse adequado. Caso contrário, o pai ficaria fora da sala até seu comportamento melhorar. Na primeira consulta, 75 crianças apresentaram comportamentos "negativos" ou "definitivamente negativos". Já na segunda consulta, 70 crianças responderam positivamente à técnica e 38 não tiveram necessidade de que o pai se retirasse, enquanto 32 apenas apresentaram sinais positivos quando o pai saiu. Os autores afirmaram então que a aplicação da técnica PPA pareceu muito bem-sucedida. 
A fim de estabelecer um programa de prevenção de cárie entre mães ansiosas e não ansiosas, Olak et al. ${ }^{14}$ analisaram 120 mães, sendo 60 mães (30 altamente ansiosas e 30 não ansiosas) usando xilitol por um mês, e mais 60 mães sem fazer $o$ tratamento. Todas as mães foram entrevistadas para saberem sobre seus hábitos de saúde bucal e educação, sendo também examinadas clinicamente. Houve ainda a participação das crianças, onde $75 \%$ delas foram examinadas, com idade de 2 a 3 anos. Os autores demonstraram que mães ansiosas escovam os dentes com menos frequência, tendo visita mais longa ao seu dentista, e um nível baixo de escolaridade em relação às não ansiosas. Porém, a ansiedade materna não teve influência na saúde bucal das crianças, pois o programa de prevenção de cárie foi eficaz tanto aos 2 como aos 3 anos de idade.

Cardoso e Loureiro ${ }^{15}$ afirmam que crianças e adultos estão suscetíveis ao estresse, e consideram que a vivência do estresse está associada à consequências negativas para a vida das pessoas. Assim sendo os autores avaliaram as manifestações de estresse que permeiam o atendimento odontológico em uma clínica-escola, e concluíram que o atendimento odontopediátrico mostrou-se permeado de manifestações de estresse por parte das crianças, acompanhantes e alunos, o que desfavorece o aprendizado por parte dos alunos. Vargas et al. ${ }^{16}$ avaliaram ainda a percepção do estudante de odontologia sobre os fatores estressores relacionados ao atendimento odontológico infantil, concluindo que todos os fatores relacionados ao paciente infantil foram considerados estressores, principalmente os associados ao comportamento da criança.

Reis $^{17}$ realizou estudo onde concluiu que é imprescindível conhecer o paciente infantil e analisar todas as variáveis que determinam seu comportamento: gênero, idade, relacionamento com os pais, grau de maturidade e experiências médico-odontológicas anteriores. Costa et al. ${ }^{18}$ acreditam que quando a criança manifesta recusa em participar do tratamento odontológico, o princípio ético que deve nortear a decisão pelo uso de técnicas restritivas deve ser o da beneficência: "estaremos fazendo o bem e evitando o mal?". Nessa situação, o cirurgião-dentista deve olhar para além do benefício odontológico e também pensar no cuidado à criança em sua totalidade.

Segundo as pesquisas de Brandenburg et al. ${ }^{19}$, ao tratar da promoção da saúde infantil, da prevenção de doenças ou da adesão ao tratamento, é necessário que o profissional compreenda o papel dos pais e busque reeducar o comportamento dos mesmos para que possa atingir objetivos que tragam benefícios para a criança. Os autores afirmam também que a forma como a criança reage no dentista pode depender, entre outros fatores, de sua relação como os pais, que ensinam diferentes repertórios necessários para enfrentar ou não um atendimento odontológico.

Felix et al. ${ }^{20}$ afirmam que o medo faz parte do desenvolvimento do ser humano, podendo causar grande ou pequena influência sobre o dia a dia do indivíduo. Em crianças, o medo faz parte das descobertas e amadurecimento. Por outro lado, esse medo pode causar traumas por toda uma vida, o que pode ocorrer, por exemplo, com o medo de ir a consultórios odontológicos. Costa et al. ${ }^{18}$ afirmam que os direitos estabelecidos no Estatuto da Criança e do Adolescente (ECA) deverão ser respeitados pela família, pela sociedade e pelo Estado, sob pena de responderem pelos danos causados. Os autores ainda relatam que o Art. 18 evidencia que todos devem velar pela dignidade da criança e do adolescente, pondo-os a salvo de qualquer tratamento desumano, violento, aterrorizante, vexatório ou constrangedor.

No estudo de Leite et al. ${ }^{21}$ é possível observar que o paciente infantil precisa ser considerado como um todo, para que a ansiedade e a tensão emocional infantil não comprometam o tratamento. Segundo os autores é necessário amar o paciente infantil, fazer-se gostar por ele, gostar de tratá-lo, ter paciência, bom senso, capacidade de persuasão, aptidão e capacidade de manter diálogos, pois a comunicação entre profissional e paciente é essencial para o sucesso do tratamento em paciente infantil com medo e ou ansiedade. Martins et al. ${ }^{22}$ (2016) partiram do pressuposto de que muitas crianças que necessitam de várias consultas de controle já efetuaram, anteriormente, tratamentos invasivos, dolorosos e desconfortáveis. Os autores reforçam, assim, a necessidade de iniciativas no âmbito da educação para a saúde bucal, de consultas de rastreio e de procedimentos de natureza preventiva, os quais poderiam evitar que a saúde bucal da criança estivesse de tal modo deteriorada, a ponto de precipitar a ocorrência de procedimentos clínicos considerados mais traumáticos.

Alguns tópicos em odontopediatria geraram mais divergência de opinião na literatura, como a dúvida de que se deve ou não incluir os pais dentro da clínica. De acordo com Nathan et al. ${ }^{23}$, para crianças em idade pré-escolar, crianças com falta de potencial cooperativo, e aqueles com uma história de experiência anterior desagradável ou com medo, a exclusão arbitrária dos pais da sala de atendimento parece mais vista como improdutiva e injustificada. Já para Felix et al. ${ }^{20}$, os responsáveis/família possuem grande influência na introdução, aumento ou redução do medo e ansiedade das crianças, devido às suas próprias experiências, podendo assim favorecer ou desfavorecer o tratamento.

Salvitti de Sá Rocha et al. ${ }^{24}$ afirmam ser necessário sistematizar procedimentos de intervenção em que seja possível avaliar não apenas os comportamentos dos pacientes, mas a interação dentista-paciente, uma vez que a utilização de estratégias de manejo pode favorecer a adaptação da criança às exigências do tratamentos. Uma estratégia de manejo do paciente e sua família foi apresentada por Stutz et al. ${ }^{25}$ em 2014, que publicaram os resultados de um projeto de extensão chamado Sala de Espera, onde estudantes interagiam com o paciente e sua família no momento prévio à assistência odontológica, visando a redução de ansiedade em relação aos procedimentos odontológicos e a melhoria das condições de saúde e higiene bucal dos pacientes por meio de processos educativos.

Encerrando esta revisão, ressalta-se o estudo de Shindova et al. ${ }^{26}$ que avaliaram o efeito da presença dos pais durante o exame clínico, sobre crianças entre 6-12 anos com medo de ir ao dentista, através da medida de parâmetros de estresse. Os graus de ansiedade foram avaliados utilizando uma combinação objetiva e subjetiva de parâmetros, incluindo frequência cardíaca, saturação de oxigênio e classificação de ansiedade. Os autores concluíram que a presença dos pais ou ausência na sala de tratamento não tem impacto sobre o nível de ansiedade de crianças durante seu exame clínico. A Tabela 1 apresenta os estudos relatados nesta revisão, por ordem alfabética, de acordo com o sobrenome do primeiro autor.

\section{DISCUSSÃO}

O medo odontológico pode ser considerado um preditor do comportamento infantil ${ }^{4}$. Assim sendo, 
Losso et al. ${ }^{5}$ sugerem que estratégias para a avaliação, prevenção e controle da ansiedade devam ser implementadas para permitir um melhor tratamento para as crianças, adolescentes e seus pais.

Tabela 1. Estudos sobre a influência da presença e do comportamento das mães na sala de atendimento sobre o comportamento de seus filhos

\begin{tabular}{|c|c|c|c|}
\hline Autores & Ano & Objetivo & Conclusão \\
\hline Afshar et al. ${ }^{1}$ & 2011 & $\begin{array}{l}\text { Avaliar o comportamento de crianças iranianas de } 5 \text { anos, } \\
\text { incluindo a ansiedade e cooperação em relação à presença } \\
\text { dos pais na primeira e segunda visita ao dentista }\end{array}$ & $\begin{array}{l}\text { A presença ou ausência dos pais não afeta a ansiedade } \\
\text { dessas crianças de } 5 \text { anos de idade }\end{array}$ \\
\hline Araújo et al. $^{\prime}$ & 2010 & $\begin{array}{l}\text { Conhecer a opinião dos pais a respeito da sua permanência } \\
\text { junto à criança durante o atendimento odontológico }\end{array}$ & $\begin{array}{c}\text { A maioria dos pais prefere estar presente durante o } \\
\text { atendimento odontológico, justificando que a criança se } \\
\text { sentiria mais segura }\end{array}$ \\
\hline Beaton et al. $^{2}$ & 2013 & $\begin{array}{c}\text { Explorar a literatura para responder à pergunta: "Por que as } \\
\text { pessoas têm medo do dentista?" }\end{array}$ & $\begin{array}{l}\text { Os resultados mostram que existem implicações práticas e } \\
\text { claras indicadas pela pesquisa existente nesta área: uma } \\
\text { melhor compreensão do medo dental, ansiedade e fobia } \\
\text { pode evitar a evasão tratamento } \\
\end{array}$ \\
\hline Brandenburg et al. ${ }^{3}$ & 2009 & $\begin{array}{l}\text { Apresentar uma descrição dos processos de interação que } \\
\text { podem ocorrer no ambiente Odontopediátrico }\end{array}$ & $\begin{array}{c}\text { Existem alternativas para que tanto a criança quanto o } \\
\text { odontopediatra sejam beneficiados, sem que se tenha que } \\
\text { apelar para a contenção física }\end{array}$ \\
\hline Brandenburg et al. $^{19}$ & 2013 & $\begin{array}{l}\text { Investigar o comportamento da mãe como uma das variáveis } \\
\text { controladoras do comportamento infantil de não colaboração }\end{array}$ & $\begin{array}{l}\text { Pais podem ser instruídos e treinados para agirem de } \\
\text { forma mais adequada durante o atendimento odontológico, } \\
\text { em benefício da saúde de seu filho }\end{array}$ \\
\hline Cadermatori $^{4}$ & 2014 & $\begin{array}{c}\text { Avaliar o comportamento infantil durante visitas } \\
\text { odontológicas }\end{array}$ & $\begin{array}{l}\text { A maioria das crianças de } 7 \text { a } 13 \text { anos apresentou } \\
\text { comportamento positivo. Porém, a complexidade foi } \\
\text { deixando o comportamento cada vez mais negativo }\end{array}$ \\
\hline Cardoso e Loureiro $^{15}$ & 2008 & $\begin{array}{l}\text { Caracterizar os problemas comportamentais das crianças } \\
\text { atendidas e as manifestações de estresse das crianças, dos } \\
\text { seus acompanhantes e dos alunos de odontologia }\end{array}$ & $\begin{array}{c}\text { Constatou-se que o atendimento odontopediátrico mostrou- } \\
\text { se permeado de manifestações de estresse por parte das } \\
\text { crianças, acompanhantes e alunos, condição pouco } \\
\text { favorecedora }\end{array}$ \\
\hline Colares et al. ${ }^{T 1}$ & 2013 & $\begin{array}{l}\text { Avaliar a ansiedade, dor e / ou desconforto relacionado à } \\
\text { tratamento dentário em crianças de } 5 \text { a } 12 \text { anos de idade }\end{array}$ & $\begin{array}{c}\text { Uma elevada percentagem de crianças apresentaram } \\
\text { ansiedade e dor }\end{array}$ \\
\hline Coric et al. ${ }^{10}$ & 2014 & $\begin{array}{l}\text { Preencher uma ficha de conhecimento, estudando DFA } \\
\text { (medo dental e ansiedade) em crianças mais velhas e seus } \\
\text { pais com medidas validadas }\end{array}$ & $\begin{array}{c}\text { Há evidências da coexistência de medo de dentista nos } \\
\text { pais e crianças mais velhas. Estes resultados podem } \\
\text { ajudar a desenvolver intervenções que irão prevenir ou } \\
\text { aliviar DFA infantil }\end{array}$ \\
\hline Costa et al. $^{18}$ & 2008 & $\begin{array}{c}\text { Consiste no levantamento e análise de aspectos éticos e } \\
\text { legais envolvidos no emprego da técnica de separação } \\
\text { durante o manejo comportamental da criança em situação } \\
\text { de atendimento odontológico }\end{array}$ & $\begin{array}{l}\text { Não há evidências de que a separação da criança de seu } \\
\text { acompanhante, visando o melhor comportamento da } \\
\text { primeira, produza algum efeito }\end{array}$ \\
\hline Felix et al. ${ }^{20}$ & 2016 & $\begin{array}{l}\text { Verificar e refletir se existe influência do medo e da } \\
\text { ansiedade no comportamento negativo de indivíduos de } \\
\text { idades diversas, diante do tratamento odontológico }\end{array}$ & $\begin{array}{l}\text { Percebeu-se que os aspectos que influenciam são } \\
\text { multifatoriais e que os responsáveis/família detém grande } \\
\text { participação no comportamento adquirido }\end{array}$ \\
\hline Goettems et al. $^{6}$ & 2011 & $\begin{array}{c}\text { Este estudo teve como objetivo avaliar a influência de } \\
\text { comportamento maternal relacionados à ansiedade dental, } \\
\text { na experiência de cárie dentária da criança }\end{array}$ & $\begin{array}{c}\text { Este estudo mostrou que a idade, sexo e renda familiar } \\
\text { determinada pelo CPOD foram médios, enquanto a } \\
\text { prevalência de cárie foi influenciada por idade, sexo e nível } \\
\text { de ansiedade dental materna }\end{array}$ \\
\hline Khawja et al ${ }^{9}$ & 2015 & $\begin{array}{l}\text { Descrever os efeitos de uma preparação por simulação } \\
\text { sobre o comportamento de crianças com histórico de não } \\
\text { colaboração, submetidas a tratamento odontológico }\end{array}$ & $\begin{array}{l}\text { O procedimento preparatório foi eficaz para o ensino de } \\
\text { respostas de enfrentamento, e a utilização destas } \\
\text { estratégias pode favorecer a adaptação da criança às } \\
\text { exigências do tratamento }\end{array}$ \\
\hline Kotsanos et al. $^{13}$ & 2009 & $\begin{array}{c}\text { Fazer a técnica de PPA (pais presentes/ ausentes), a fim de } \\
\text { gerenciar o comportamento da criança não colaboradora, } \\
\text { onde se a criança não colaborasse o pai ou responsável iria } \\
\text { se retirar da sala durante o atendimento }\end{array}$ & A aplicação da técnica PPA parece muito bem sucedida \\
\hline Leite et al. ${ }^{21}$ & 2013 & $\begin{array}{l}\text { Abordar aspectos que norteiam o atendimento odontológico } \\
\text { do paciente infantil com medo e ou ansiedade em saúde } \\
\text { pública, apresentando formas de condução psicológica }\end{array}$ & $\begin{array}{c}\text { O medo e ou ansiedade do paciente infantil ao tratamento } \\
\text { odontológico pode ser revertido, se o atendimento for bem } \\
\text { conduzido psicologicamente, considerando o perfil da } \\
\text { criança e de seus responsáveis }\end{array}$ \\
\hline Losso et al. ${ }^{5}$ & 2013 & $\begin{array}{c}\text { Comparar a ansiedade dentária em crianças, adolescentes e } \\
\text { seus pais }\end{array}$ & $\begin{array}{c}\text { Um grau moderado de ansiedade dental foi predominante } \\
\text { entre as crianças, adolescentes e pais que participaram do } \\
\text { inquérito }\end{array}$ \\
\hline Martins et al. ${ }^{22}$ & 2016 & $\begin{array}{l}\text { Avaliar a existência de contágio emocional de ansiedade no } \\
\text { par relacional encarregado de educação/criança. }\end{array}$ & $\begin{array}{c}\text { Apesar de não se verificar a existência de contágio } \\
\text { emocional para a amostra estudada, é inegável a } \\
\text { existência da problemática da ansiedade }\end{array}$ \\
\hline Nathan et al. ${ }^{23}$ & 2015 & $\begin{array}{c}\text { Explorar as visões contemporâneas de odontopediatras e } \\
\text { pais em relação à inclusão ou exclusão dos pais tem um } \\
\text { impacto sobre o comportamento das crianças e resposta ao } \\
\text { tratamento odontológico }\end{array}$ & $\begin{array}{c}\text { Para crianças em idade pré -escolar, crianças não- } \\
\text { colaboradoras, e aqueles com uma história de experiência } \\
\text { anterior com medo ou ansiedade, a exclusão arbitrária dos } \\
\text { pais durante o tratamento odontológico aparece cada vez } \\
\text { mais visto como improdutivo e injustificada }\end{array}$ \\
\hline Olak et al. ${ }^{14}$ & 2012 & $\begin{array}{l}\text { O objetivo deste estudo foi afim de fazer um programa de } \\
\text { prevenção de cárie entre as mães ansiosas e não-ansiosas }\end{array}$ & $\begin{array}{c}\text { Todas as crianças foram beneficiadas no programa de } \\
\text { prevenção de cárie a base de xilitol independente da } \\
\text { ansiedade materna }\end{array}$ \\
\hline Peretz et al. ${ }^{12}$ & 2004 & $\begin{array}{c}\text { O objetivo do presente estudo foi investigar a ansiedades } \\
\text { dental das crianças, pais e cuidadores de estudantes em um } \\
\text { ambiente escolar }\end{array}$ & $\begin{array}{c}\text { Ansiedade dental dos estudantes, ou a sua ansiedade } \\
\text { antes de serem tratadas, pode não estar diretamente } \\
\text { relacionado com a ansiedade odontológica. No entanto, } \\
\text { uma correlação positiva existente entre pais e ansiedade } \\
\text { dental infantil }\end{array}$ \\
\hline Reis $^{17}$ & 2011 & $\begin{array}{c}\text { Avaliar, comparativamente, as reações emocionais } \\
\text { (ansiedade e medo) de crianças com e sem experiência } \\
\text { prévia aos procedimentos odontológicos }\end{array}$ & $\begin{array}{l}\text { As expectativas dos pais/responsáveis quanto às reações } \\
\text { das crianças foram boas, sugerindo que a concepção } \\
\text { positiva do atendimento odontológico }\end{array}$ \\
\hline Salvitti de Sá Rocha et al. ${ }^{24}$ & 2015 & $\begin{array}{l}\text { Descrever os efeitos de uma preparação por simulação } \\
\text { sobre o comportamento de crianças com histórico de não } \\
\text { colaboração, submetidas a tratamento odontológico }\end{array}$ & $\begin{array}{c}\text { O procedimento preparatório foi eficaz para o ensino de } \\
\text { respostas de enfrentamento, e a utilização destas } \\
\text { estratégias pode favorecer a adaptação da criança às } \\
\text { exigências do tratamento }\end{array}$ \\
\hline Shindova et al. ${ }^{26}$ & 2013 & $\begin{array}{c}\text { Avaliar o efeito da presença dos pais sobre a ansiedade em } \\
\text { crianças de 6-12 anos durante o exame clínico com medição } \\
\text { subjetiva e parâmetros objetivos de estresse }\end{array}$ & $\begin{array}{l}\text { A presença dos pais ou ausência na sala de tratamento } \\
\text { não tem nenhum impacto sobre a ansiedade nível das } \\
\text { crianças de 6-12 anos durante a sua clínica exame }\end{array}$ \\
\hline Stutz et al. ${ }^{25}$ & 2014 & $\begin{array}{c}\text { Mobilizar e despertar a clientela atendida na clínica do } \\
\text { Hospital Odontológico/FOUFU quanto aos cuidados para } \\
\text { uma saúde bucal adequada }\end{array}$ & $\begin{array}{c}\text { Os resultados obtidos demonstram a importância do } \\
\text { desenvolvimento desse tipo de projeto de extensão na } \\
\text { promoção da saúde e do bem-estar }\end{array}$ \\
\hline Tomita et al. $^{8}$ & 2007 & $\begin{array}{l}\text { Analisar os comportamentos de dez mães durante sessões } \\
\text { sequenciais de atendimento odontológico de seus filhos }\end{array}$ & $\begin{array}{l}\text { Algumas mães demonstram maior nível de ansiedade do } \\
\text { que outras, mas existem certos comportamentos que são } \\
\text { comuns a todas elas }\end{array}$ \\
\hline Vargas et al. ${ }^{16}$ & 2013 & $\begin{array}{c}\text { Avaliar a percepção do estudante de odontologia sobre os } \\
\text { fatores estressores relacionados ao atendimento } \\
\text { odontológico infantil }\end{array}$ & $\begin{array}{c}\text { Todos os fatores relacionados ao paciente infantil foram } \\
\text { considerados estressores, principalmente os associados ao } \\
\text { comportamento da criança }\end{array}$ \\
\hline
\end{tabular}


Para os autores é possível realizar uma avaliação simples e eficiente da ansiedade na rotina de uma clínica odontológica pediátrica com o uso de instrumentos validados. Os autores afirmam que uma abordagem adequada sobre crianças e adolescentes com ansiedade odontológica pode ajudar a estabelecer uma boa experiência odontológica e uma relação de confiança entre os odontopediatras, o paciente e os pais.

Cadermatori ${ }^{4}$ acredita também que na odontopediatria os especialistas têm que ter medidas preventivas e habilidades para a realização do tratamento odontológico, além de estarem preocupados também com o bem-estar da criança ou adolescente durante o procedimento. Para Stutz et al. $^{25}$, um tratamento sensível às questões emocionais dos pacientes envolvidos pode gerar bem-estar à medida que reduz a ansiedade característica que

permeia a atuação clínica em odontologia.

Segundo Felix et al. $^{20}$, os responsáveis/família possuem grande influência na introdução, aumento ou redução do medo e ansiedade das crianças, devido às suas próprias experiências. Reconhecer o estresse do paciente e da família é importante para que o profissional possa instruir os pais e encaminhar a criança para receber os cuidados necessários, o que facilitará a própria rotina de atendimento, podendo o profissional lidar mais facilmente com as situações adversas, sem aumentar o estresse existente ${ }^{4}$.

Em relação à condição socioeconômica, Goettems et al. ${ }^{6}$ observaram que pessoas de baixa renda familiar têm mais ansiedade e índice de cárie, corroborando com os resultados de Khawja et al. ${ }^{9}$ que também relataram grande associação entre renda familiar baixa e grande ansiedade materna, juntamente com a cárie infantil.

Ainda sobre a influência da ansiedade dos pais sobre o comportamento das crianças durante a assistência odontológica, Coric et al. ${ }^{10}$ afirmam que os pais têm grande efeito no DFA (associação entre o medo de dentista e ansiedade) de uma criança. No entanto, Olak et al. ${ }^{14}$ concluíram que a ansiedade materna não teve influência na saúde bucal das crianças, pois o programa de prevenção de cárie foi eficaz para elas.

Brandenburg et al. ${ }^{3}$ acreditam que há técnicas que possam diminuir a ansiedade odontológica. A técnica PPA (pais presentes/ausentes) de Kotsanos et al. ${ }^{13}$ foi muito bem sucedida, como relatado anteriormente. Araújo et al. ${ }^{7}$ relataram que os pais gostam de permanecer na sala durante $o$ atendimento, dizendo que a criança estaria mais segura. No relato de Tomita et al. ${ }^{8}$ as mães preferiam ficar segurando a mão da criança, ou até mesmo conter e observar durante o tratamento odontológico.

Salvitti de Sá Rocha et al. ${ }^{24}$ inferem a necessidade de novas investigações para a sistematização do uso de estratégias de manejo do comportamento e de procedimentos de preparação para rotinas invasivas em Odontopediatria. Lembrando que tanto os pais que preferem estar presentes durante $o$ atendimento, quanto os que preferem aguardar na sala de espera justificaram da mesma forma sua preferência, que a criança ficaria mais segura ${ }^{7}$, a presença/ausência dos pais durante $\mathrm{o}$ atendimento odontológico da criança deveria/poderia também ser decidida com base em protocolos sistematizados, visando o bem estar da criança, familiares e equipe odontopediátrica.

\section{CONCLUSÃO}

Seria favorável que o paciente infantil fizesse a primeira visita ao consultório odontológico o mais cedo possível, quando ainda não houvesse a necessidade de procedimentos invasivos, cedendo ao profissional a oportunidade de realizar prevenção em saúde bucal e o condicionamento da criança, para que ela se acostume com o ambiente odontológico, com os procedimentos, e com a equipe. Não sendo possível o atendimento precoce, o medo e a ansiedade devem ser controlados por métodos e técnicas de manejo do comportamento, sendo indispensável ainda o diálogo e a participação sistematizada dos pais, proporcionando uma adequada relação do profissional com o paciente e sua família. A presença das mães não é o principal fator influenciador no comportamento inadequado da criança dentro do consultório odontológico. Com a orientação adequada do profissional, ela pode auxiliar no manejo do comportamento de sua criança, dentro ou fora da sala de atendimento. Isso será possível após o estabelecimento de vínculo de confiança tanto entre família-profissional, quanto da criança com o seu dentista, tornando possível uma assistência odontológica eficaz e segura para a criança e a equipe.

\section{REFERÊNCIAS}

1. Afshar H, Nakhjavani YB, Mahmoudi-Gharaei J, Paryab M, Zadhoosh S. The effect of Parental Presence on the 5 year- Old Children's Anxiety and Cooperative Behavior in the First and Second Dental Visit. Iran J Pediatr. 2011; 21(2):193-200

2. Beaton A, Freeman R, Humphris G. Why Are People Afraid of the Dentist? Med Princ Pract. 2014; 23(4):295301.

3. Brandenburg OJ; Haydu V.B. Contribuições da Análise do Comportamento em Odontopediatria. Psicol cienc prof. 2009; 29(3):462-75.

4. Cadermatori. Comportamento infantil durante consultas odontológicas sequenciais: influência de características clínicas, psicossociais e maternas. Universidade Federal de Pelotas / Sistema de Bibliotecas Catalogação na Publicação 2014.

5. Losso E, Assunção C, Andreatini R, Menezes JV. The retationship between dental anxiety in children, adolescents and their parents at dental environment. J Indian Soc Pedod Prev Dent. 2013;31(3):175-9.

6. Goettems ML, Ardenghi TM, Romano AR, Demarco FF, Torriani DD. Influence of Maternal Dental Anxiety on the Child's Dental Caries Experience. Caries Res 2012; 46(1):3-8.

7. Araújo SM, Silveira EG, Mello LD, Caregnato M, DaL'Asta VG. Ponto de vista dos pais em relação a sua presença durante o atendimento odontológico de seus filhos. Salusvita. 2010; 29(2):17-27

8. Tomita LM, Costa Junior AL, Moraes ABA. Ansiedade materna manifestada durante o tratamento odontológico de seus filhos. Psico-USF 2007; 12(2):249-56.

9. Khawja SG, Arora R, Shah AH, Wyne AH, Sharma A. Maternal dental anxiety and its Effect on Caries Experience Among Children in Udaipur. India. J Clin Diagn Res. 2015; 9(6):ZC42-5.

10. Coric A, Banozic A, Klaric M, Vukojevic K, Puljak L. Dental fear and anxiety in older children: an association with parental dental anxiety and effective pain coping strategies. J Pain Res. 2014; 7:515-21.

11. Colares V, Franca C, Ferreira A, Amorim Filho HA, Oliveira MC. Dental anxiety and dental pain in 5- to 12year-old children in Recife, Brazil. Eur Arch Paediatr Dent. 2013; 14(1):15-9.

12. Peretz B, Nazarian Y, Bimstein E. Dental anxiety in a student's paediatric dental clinic: children, parents and students. Int J Paediatr Dent. 2004; 14(3):192-8. 
13. Kotsanos N, Coolidge T, Velonis D, Arapostathis KN Short communication: A form of 'parental presence/ absence' (PPA) technique for the child patient with dental behavior management problems. Eur Arch Paediatr Dent. 2009; 10(2):90-2.

14. Olak J, Saag M, Vahlberg T, Söderling E, Karjalainen S. Caries prevention with xylitol lozenges in children related to maternal anxiety. Eur Arch Paediatr Dent. 2012; 13(2):64-9.

15. Cardoso CL, Loureiro SR. Estresse e comportamento do colaboração em face do tratamento odontopediátrico. Psicol estud. 2008;13(1):133-41.

16. Vargas NA, Fontes BNV, Tolentino AB, Rodrigues LCM, Oliveira FS, Castro AM. Percepção do estudante de Odontologia sobre os fatores estressores relacionados ao atendimento infantil. Faculdade de Odontologia de Lins/ Unimep 2013; 23: 11-19.

17. Reis JR. Avaliação de reações emocionais em odontopediatria [dissertação]. Brasília: Universidade de Brasília; 2011.

18. Costa L, Martorell LB, Azevedo AAC, Prado MM Legitimidade e licitude da técnica de separação acompanhante-criança durante $\mathrm{o}$ atendimento odontológico no contexto brasileiro. Pesqui bras odontopediatria clin integr. 2008; 8(3):367-73.

19. Brandenburg OJ, Marinho-Casanova ML. Relação mãecriança durante $\mathrm{o}$ atendimento odontológico: contribuições da análise do comportamento. Estud psicol. 2013; 30(4):629-40.

20. Felix LF, Brum SC, Barbosa CCN, Barbosa O. Aspectos que influenciam nas reações comportamentais de crianças em consultórios odontológicos. R Pró-Uni. 2016; 7(2):13-6.

21. Leite DFBM, Muniz IAF, Farias IAP. Condução psicológica do paciente infantil em Saúde Pública. Odontol Clin Cient. 2013; 12(4):251-4.

22. Martins N, Dias MR. Contágio emocional de ansiedade encarregado de educação/ criança em odontopediatria. Rev Port Estomatol Med Dent Cir Maxilofac. 2016;57(3):164-70.

23. Nathan JE, Rayman MS, Golden BE, Vargas KG. Discretionary parental presence in the dental operatory: a survey of pediatric dentists and parents. Pediatr Neonatal Nurs Open J. 2015; 2(2):50-61.

24. Salvitti Sá Rocha RA, Rolim GS, Moraes ABA. Procedimento preparatório para atendimento de pacientes não colaboradores em odontopediatria. Acta Comportamentalia. 2015; 23(4):423-35.

25. Stutz BL, Mendonça LC, Gomes SSR. Sala de espera: um suporte ao atendimento odontológico e à saúde bucal. J Manage Prim Health Care. 2014; 5(1):54-61.

26. Shindova M, Belcheva A. The effect of parental presence on the dental anxiety during clinica exatamination in children aged 6-12 years. J IMAB. 2013; 19(4):435-8.

\section{CONFLITO DE INTERESSES}

Os autores declaram não haver conflitos de interesse.

\section{AUTOR PARA CORRESPONDÊNCIA}

Adriana Sales Cunha-Correia

dricunhacorreia@yahoo.com.br

Submetido em 30/10/2016 Aceito em 05/12/2016 